\title{
El síndrome del ATRA como complicación del tratamiento en la leucemia promielocítica aguda
}

\author{
M. MARTÍN DEL POZO, E. CISNEROS DE LA FUENTE*, F. SOLANO**, \\ M. L. MARTÍN**, J. DE LA SERNA** \\ Servicio Urgencias Fundación Hospital de Alcorcón, Madrid. *Servicio de Medicina \\ Interna de Hospital de Manacor, Mallorca. **Servicio de Hematología de Hospital \\ Universitario 12 de Octubre. Madrid
}

THE RETINOIC ACID SYNDROME, A COMPLICATION OF THE RAPY WITH ALL-TRANS RETINOIC ACID

\begin{abstract}
RESUMEN
Objetivo: Describir el síndrome del ATRA como complicación del tratamiento con ácido todo-trans-retinoico (ATRA) o tretinoino, en la leucemia promielocítica aguda (LPA).

Métodos: Estudio retrospectivo, incluyendo cinco pacientes diagnosticados de LPA según los criterios $\mathrm{FAB}$, que recibieron tratamiento de inducción con ATRA y desarrollaron en su evolución el Síndrome del ATRA.

Resultados: Tres pacientes fueron tratados al diagnóstico y dos en la recidiva leucémica. Todos ellos recibieron ATRA, $45 \mathrm{mg} / \mathrm{m} 2 /$ día por vía oral en pauta continua, acompañado en tres casos de quimioterapia convencional. Entre el día +1 y el día +11 desde el inicio del tratamiento, los pacientes desarrollaron fiebre, insuficiencia respiratoria con infiltrados pulmonares, ganancia de peso y edemas. El inicio del cuadro fue precedido de leucocitosis en todos los casos. Se descartaron infeccion e insuficiencia cardíaca. La clínica progresó en el contexto de amplia cobertura antibiótica. Cuatro pacientes recibieron tratamiento con dexametasona endovenosa (10 mg/12 horas), de los cuales tres evolucionaron favorablemente y uno falleció. El paciente que no se trató falleció.

Conclusión: El síndrome del ATRA es una complicación potencialmente mortal en pacientes con LPA que reciben tratamiento de inducción con ATRA. Habitualmente aparece a los pocos días del inicio del tratamiento. Ante la sospecha de este cuadro clínico debe establecerse tratamiento precoz con esteroides en dosis altas.
\end{abstract}

PALABRAS CLAVE: Síndrome del ATRA (ácido todo-trans-retinoico). Leucemia promielocítica aguda.

\section{ABSTRACT}

Objective: To describe the retinoic acid syndrome, a complication of therapy with all-trans retinoic acid (ATRA) in acute promyelocitic leu kemia (APL).

Patients and methods: Retrospective study of five patients with a morphologic diagnosis of APL by the French-American-British (FAB) classification that were treated for remission induction with ATRA and developed the ATRA syndrome.

Results: Three patients in newly diagnosed APL and two in leukemia relapse were analyzed. All patients received with ATRA $45 \mathrm{mgrs} / \mathrm{m} 2 /$ day, and three of them also received chemotherapy. Patients developed fever, respiratory distress, pulmonary infiltrates, weight gain and edemas. The onset of this symptom complex occurred from 1 to 11 days after starting tre atment and was preceded by an increased in peripheral blood leukocytes. Infections or congestive heart failure were ruled out. The clinical course progressed while patients being treated with wide spectrum antibiotics. Four patients were treated with high doses corticosteroid therapy (dexame tasone 10 mgrs intravenously every 12 hours), in three of them full recovery was attained and one died. One patient that did not received steroids died.

Conclusions: The use of all-trans retinoic acid to induce hematolo gic remission in APL patients is associated in same patients with the development of ATRA syndrome, a life threatening complication. Symp toms begin in the first days of treatment. If this syndrome is suspected, early treatment with high dose steroids should be iniciated.

KEY WORDS: Retinoic acid syndrome. Acute promyelocitic leukemia.

Martín del Pozo M, Cisneros de la Fuente E, Solano F, Martín ML, de la Serna J. El síndrome del ATRA como complicacion del tratamiento en la leucemia promielocítica aguda. An Med Interna (Madrid) 2001; 18: 195-200.

\section{INTRODUCCIÓN}

La leucemia promielocítica aguda (LPA) es el tipo de leucemia mieloide aguda más frecuente en nuestro medio. En España alcanza un $20 \%$ del total de casos de leucemia aguda del adulto, mientras que en las series anglosajonas la incidencia es menor, próxima al 10\% (1). La LPA tiene una morfología celular característica, con promielocitos atípicos hipergranulares y se asocia con frecuencia a diátesis hemorrágica y coagulopatía de consumo. Existe una variante microgranular
(LPAv) que suele cursar con leucocitosis y puede plantear dificultades en su reconocimiento morfológico. La demostración de la translocación $\mathrm{t}(15 ; 17)$ o la detección por Southernblot o RT-PCR del reordenamiento PML/RARAa, gen de fusión resultante de esta translocación, permite diferenciar la LPA de otras leucemias agudas (2).

La LPA tiene una peculiar sensibilidad al tratamiento, que ha contribuido a individualizar su manejo respecto al resto de las leucemias, la superior actividad de las antraciclinas (daunorrubicina o idarubicina) sobre otros agentes citotóxicos en la

Trabajo aceptado: 30 de Julio de 1999

Correspondencia: Dra. Mar Martín del Pozo. Puentecesures $n^{\circ}$ 1, bloq A, 5º D. 28029 Madrid. 
inducción de la remisión completa (3), y la mayor utilidad del tratamiento continuado con quimioterapia en dosis bajas que mantiene con mayor frecuencia la remisión clínica frente a otras opciones mas agresivas de tratamiento postremisión $(4,5)$. Pero es de mayor transcendencia la actividad de los retinoides, especialmente del tretinoíno o ácido todo-trans-retinoico (ATRA), que por sí solo es capaz de inducir la remisión completa en un 80-90\% de los casos de LPA de novo o en recaída (6-11), y contribuir al mantenimiento de la remisión completa (12). A diferencia de la quimioterapia convencional, el ATRA actúa a través de la inducción de la diferenciación de promielocitos y blastos a células mieloides maduras y facilitando su ulterior apoptosis (1). Esta nueva vía de tratamiento de la leucemia ha revolucionado la investigación básica y abierto el camino para la investigación de otros agentes diferenciadores e inductores de apoptosis, demostrándose recientemente que el trióxido de arsénico $\left(\mathrm{As}_{2} \mathrm{O}_{3}\right)$ posee un efecto similar en la LPA (13).

En la práctica clínica, la utilidad del ATRA se ve incrementada por su capacidad de inducir la remisión sin producir aplasia medular y mejorar rápidamente la coagulopatía de consumo, lo que contribuye a reducir la morbi-mortalidad por hemorragias o infecciones durante el tratamiento de inducción (7). Sin embargo el tratamiento con ATRA cuenta con dos importantes limitaciones: el desarrollo de resistencias a corto plazo y el llamado "Síndrome del ATRA" (SA) $(1,6)$.

La resistencia al ATRA se produce por una reducción progresiva de los niveles plasmáticos de la droga provocada por la inducción enzimática de la citocromo-oxidasa P450 y de proteínas transportadoras de membrana (CRABP) a nivel hepático, lo que determina una menor biodisponibilidad de la droga $(14,15)$. Esto supone que las remisiones completas conseguidas con ATRA recidivan en breve plazo si no se aplican otras medidas complementarias.

El SA se caracteriza clínicamente por fiebre, insuficiencia respiratoría con hipoxemia, infiltrados pulmonares en la radiología de torax, derrame pleural y ganancia de peso con edemas o hepatomegalia. Algunos pacientes también desarrollan hipotensión ortostática, derrame pericárdico, insuficiencia renal o alteraciones en pruebas de función hepática (16). Puede aparecer hasta en el $25 \%$ de los pacientes con LPA tratados con ATRA en la inducción, con o sin quimioterapia acompañante. Típicamente se inicia en los primeros días del tratamiento y sin las medidas adecuadas produce una elevada mortalidad. Sus manifestaciones pueden solaparse o confundirse con otras complicaciones como una neumonía o infección nosocomial, insuficiencia cardiaca o pericarditis $(1,2,8)$.

En el periodo comprendido entre 1993 y 1995, hemos contabilizado cinco casos de síndrome del ATRA entre trece pacientes con leucemia promielocítica, que recibieron ATRA en el tratamiento de inducción. Dadas las características del SA a su presentación y el curso clínico observado creemos que es de interés la descripción de los casos y la discusión del cuadro.

\section{CASOS APORTADOS}

Caso 1: Varón de 45 años que ingresa con hematomas y gingivorragias. La analítica de sangre mostró trombocitopenia (Plaquetas $36 \times 10^{9} / \mathrm{L}$ ), blastos circulantes y coagulopatía de consumo. La médula ósea estaba masivamente infiltrada por blastos y promielocitos atípicos hipergranulares. El cariotipo objetivó una línea celular con deleciones en los cromosomas 2 y 5 (46 XY,2q-,5q-) y el estudio por RT-PCR no detectó el reordenamiento PML-RARAa. Se trató con ATRA $\left(45 \mathrm{mg} / \mathrm{m}^{2} /\right.$ día $)$, daunorrubicina $\left(60 \mathrm{mg} / \mathrm{m}^{2} /\right.$ día x 3 días $)$ y citarabina (200mg/m²/día x 7 días). El control de la coagulopatía fué rápido. Al noveno día presentó fiebre sin focalidad infecciosa. La RX de torax fue normal y los hemocultivos y otros exámenes microbiológicos negativos. Se inició antibioterapia empírica con ceftacidima y amikacina, añadiéndose posteriormente vancomicina y anfotericina $\mathrm{B}$, aunque los hemocultivos continuaron siendo estériles y no se encontró foco infeccioso aparente. En los días sucesivos se deterioró de la función renal y se observaron alteraciones en el perfil hepático siendo la ecografía abdominal normal. Al día catorce y continuando febril, desarrolló insuficiencia respiratoría hipoxémica, signos radiológicos de edema pulmonar y aumento de peso. No había alteraciones electrocardiográficas y el ecocardiograma 2D fue normal. Al día siguiente un derrame pleural derecho se hizo evidente. Se suspendió el tratamiento con antibióticos nefrotóxicos y comenzó con dexametasona iv (10mg/12 horas). La fiebre desapareció de inmediato y se produjo una mejoría clínica, radiológica y gasométrica en 48 horas. Cuatro días después comenzó la recuperación hemoperiférica y el paciente fue dado de alta en remisión completa al día 28 del inicio del tratamiento. El tratamiento con esteroides se mantuvo 10 días.La función pulmonar, renal y hepática al alta eran normales. En la evolución posterior del paciente se detectó el reordenamiento del gen PML-RARAa mediante RT-PCR.

Caso 2: Mujer de 62 años con antecedente de carcinoma ductal infiltrante de mama izquierda (T2,N1,M0) tratado con tumorectomía, linfadenectomía axilar, radioterapía y tamoxifeno. Dos años después ingresó por diátesis hemorrágica. La analítica mostró leucopenia con blastos circulantes, trombocitopenia y coagulopatía de consumo. La médula osea presentaba una infiltración masiva por promielocitos atípicos. La citogenética fue $46 \mathrm{XX}, \mathrm{t}(15 ; 17)$ y se detectó el reordenamiento PML-RARA $\alpha$. Se estableció tratamiento con ATRA ( $45 \mathrm{mg} / \mathrm{m}^{2} /$ día), y dos días después, ante el desarrollo de leucocitosis, se añadieron daunorrubicina $\left(60 \mathrm{mg} / \mathrm{m}^{2} /\right.$ día x 3 días $)$ y citarabina ( $200 \mathrm{mg} / \mathrm{m}^{2} /$ día x 7 días). Al tercer día la paciente desarrolló fiebre y disnea. Presentaba además ganancia de peso y edemas sin fovea, pero la presión venosa central era normal. La radiología de torax mostraba un infiltrado alveolo-intersticial bilateral y derrame pleural bilateral. Los hemocultivos fueron estériles y un ecocardiograma -2D mostró una función cardíaca conservada. Se administraron diuréticos y antibioterapia empírica con imipenem y vancomicina sin respuesta favorable. Dos días después, ante la progresión del cuadro pulmonar se inició tratamiento con dexametasona iv (10mg/12 horas), manteniendo las dosis de ATRA y quimioterapia. En los días sucesivos y de manera progresiva desapareció la fiebre y mejoró la clínica respiratoria y la radiología pulmonar en 7 días. Se mantuvo es tratamiento con dexametasona durante 10 días. Fue dada de alta a los 40 días, en remisión completa y sin secuelas aparentes.

Caso 3: Varón de 16 años que ingresó con gingivorragias, blastosis en sangre, trombopenia, y coagulopatía de consumo, siendo diagnosticado de LPA por una médula ósea con infiltración difusa por promielocitos atípicos. El cariotipo fue normal y el reordenamiento PML-RARAa al diagnóstico negativo, aunque en la evolución posterior sí resultó positivo. Se inició tratamiento antileucémico con ATRA oral $\left(45 \mathrm{mg} / \mathrm{m}^{2} /\right.$ día). A los dos días el paciente desarrolló fiebre y tendencia a leucocitosis por lo que se asoció quimioterapia con daunorrubicina $\left(60 \mathrm{mg} / \mathrm{m}^{2} /\right.$ día x 3 días) y citarabina $\left(200 \mathrm{mg} / \mathrm{m}^{2} /\right.$ día $\mathrm{x} 7$ días) y tratamiento antibiótico empírico con imipenem y amikacina. El cuadro progresó rápidamente, con aparición de insuficiencia respiratoria grave y hemoptisis moderada. La Radiografia de tórax mostró la aparición de infiltrados algodonosos bilaterales. En las horas siguientes desarrolló fracaso hemodinámico con hipoxemia y acidosis severas, precisando ventilación mecánica y tratamiento con drogas vasoactivas para estabilizar su situación. Posteriormente presentó varios episodios de sangrado masivo por tubo endotraqueal y sonda nasogástrica, y dado que la coagulopatía de consumo continuaba activa, fue necesaria la transfusión de múltiples concentrados de plaquetas, hematíes y plasma. A la vista de la mala evolución del paciente 
con las medidas de soporte intensivo, al cuarto día de su ingreso se añadió al tratamiento dexametasona $10 \mathrm{mg} / 12$ horas. La mejoría fue evidente en 24 horas. El paciente pudo ser extubado cuatro días después, encontrándose afebril y en aplasia, pero con coagulopatía de consumo controlada. Todos los estudios microbiológicos de sangre y secreciones respiratorias fueron negativos. El tratamiento con dexametasona se mantuvo 10 días. El paciente alcanzó la remisión completa y tras el alta médica la función cardiorespiratoria era normal.

Caso 4: Mujer de 58 años con antecedente de carcinoma ductal infiltrante de mama izquierda $(\mathrm{T} 1, \mathrm{~N} 1, \mathrm{M} 0)$ que se trató con mastectomía radical, 6 ciclos de quimioterapia, radioterapia y tamoxifeno de mantenimiento. Ingresó 2 años después con leucocitosis importante, coagulopatía de consumo y una infiltración masiva de médula ósea por promielocitos microgranulares. Se diagnosticó de LPA. El cariotipo medular mostró una línea celular con 46XX 17p-, pero no pudo demostrarse el reordenamiento PML-RARAa. Recibió tratamiento inicial con quimioterapia convencional (daunorrubicina y citarabina), se consiguió la remisión completa y recibió tratamiento de consolidación con el mismo esquema. En esta fase presentó complicaciones infecciosas graves, quedando como secuela insuficiencia respiratoria moderada. A los 14 meses se demostró recidiva leucémica, con un $85 \%$ blastos en médula de morfología similar a la del diagnóstico. Esta vez se trató con ATRA oral, $45 \mathrm{mg} / \mathrm{m}^{2} /$ día y hemoterapia de soporte.

En los días siguientes comenzó con febrícula, ganancia de peso y leucocitosis progresiva hasta $23,5 \times 10^{9} / \mathrm{L}$ sin corrección de la coagulopatía.

La radiología de torax no fue expresiva y los hemocultivos resultaron negativos. Se estableció tratamiento antibiótico de amplio espectro al cuarto día sin mejorar el cuadro. Al séptimo día, bruscamente, aumentó la disnea y se observo hipoxemia severa con progresión de la radiología pulmonar, por la aparición de infiltrados alveolares bilaterales. No había cardiomegalia. La presión venosa central era normal y un ECG y una ecografía -2D descartaron pericarditis y fallo ventricular, sin mostrar signos de tromboembolismo pulmonar. Se procedió a una mayor restricción de volumen, tratamiento diurético y oxigenoterapia, pero en las horas siguientes presentó una hemiparesia izquierda, coma y exitus en parada respiratoria.
Caso 5: Varón de 54 años diagnosticado de LPA por el hallazgo de anemia y trombocitopenia. Presentaba una infiltración medular por un $76 \%$ de promielocitos atípicos, un cariotipo con una línea celular 46XX 17p- y reordenamiento PML-RARAa positivo. Recibió tratamiento de inducción con daunorrubicina y citarabina en pauta $3+7$, consolidación con citarabina a dosis intermedias y mitoxantrona, e intensificación con autotransplante de médula ósea tras quimioterapia mieloablativa con busulfan $(16 \mathrm{mg} / \mathrm{kg})$ y ciclofosfamida $(120 \mathrm{mg} / \mathrm{kg})$. En el periodo pos-transplante presentó un hemopericardio con taponamiento cardíaco que se resolvió sin secuelas con drenaje y esteroides. A los 14 meses presentó recidiva leucémica, que se manifestó con pancitopenia y coagulopatía de consumo. La médula ósea estaba infiltrada masivamente por promielocitos atípicos. Antes del tratamiento se realizó un ecocardiograma 2D que mostró una función cardíaca normal, con fracción de eyección del ventrículo izquierdo de $57 \%$. Se trató con ATRA oral $\left(45 \mathrm{mg} / \mathrm{m}^{2} / \mathrm{d}\right.$ áa). Rápidamente se observó una mejoría de la coagulopatía y un descenso de los blastos en sangre. A los siete días comenzó con fiebre, documentándose en hemocultivo P. Seudomo $n a$ aeruginosa. Se estableció tratamiento antibiótico con ceftazidima y amikacina con buena respuesta.

Tres días después comenzó con diarrea e hiperbilirrubinemia, añadiéndose vancomicina iv y metronidazol oral, interrumpiéndose la administración de ATRA al día doce.

Al día catorce comenzó nuevamente con fiebre, disnea y dolor torácico. La presión venosa central era normal. En la radiografía de torax se hizo patente un patrón alveolo-intersticial bilateral de predominio central, sin derrame pleural y con silueta cardíaca no aumentada. Un ecocardiograma -2D mostró un mínimo derrame pericárdico con función ventricular conservada. Se tomaron nuevos cultivos de sangre y secreciones respiratorias, que resultaron negativos, y se administró dexametasona (10mg/12 horas), pero la insuficiencia respiratoria progresó, apareció hemoptisis y en la radiología los infiltrados se generalizaron por ambos campos. Las medidas de soporte no fueron eficaces y el paciente desarrolló fracaso hemodinámico y posteriormente fallo multiorgánico falleciendo en 48 horas. No se concedió la necropsia.

TABLA I

CARACTERÍSTICAS CLÍNICAS DE LOS PACIENTES CON APL

\begin{tabular}{|c|c|c|c|c|c|}
\hline & Paciente 1 & Paciente 2 & Paciente 3 & Paciente 4 & Paciente 5 \\
\hline Edad (años) & 45 & 62 & 15 & 58 & 54 \\
\hline Sexo & Varón & M ujer & Varón & Mujer & Varón \\
\hline Antecedentes & No & Cáncer de mama & No & Cáncer de mama & No \\
\hline Tipo FAB & M3 & M 3v & M3 & M 3v & M3 \\
\hline $\begin{array}{l}\text { Leucocitos } \\
\left(\times 10^{6} / \mathrm{mL}\right)\end{array}$ & 5,41 & 3,7 & 9,01 & 21,6 & 8,8 \\
\hline $\begin{array}{l}\text { Blastos en } \\
\text { médula (\%) }\end{array}$ & 90 & 90 & 95 & 85 & 76 \\
\hline CID* & $\mathrm{Si}$ & $\mathrm{Si}$ & $\mathrm{Si}$ & $\mathrm{Si}$ & No \\
\hline Citogenética & $\begin{array}{l}46 X Y / 46 X Y 2 q-1 \\
46 X Y 5 q-\end{array}$ & $\begin{array}{c}\text { 46XX/46XX t }(15 ; 17) / \\
\text { 46XX y otras delecciones }\end{array}$ & $46 X Y$ & 46XX/46xx17p- & $46 X Y / 46 X Y$ \\
\hline $\begin{array}{l}\text { Inmuno } \\
\text { fenotipo }\end{array}$ & $\begin{array}{c}\text { CD45+,CD33+, } \\
\text { CD13+ } \\
\text { CD15+,CD34t }\end{array}$ & $\begin{array}{c}\text { CD45+,CD33+,CD13+, } \\
\text { CD19+,CD15-,CD11-,CD22- }\end{array}$ & $\begin{array}{l}\text { CD33+,CD13t, } \\
\text { CD2t,CD19+, } \\
\text { CD11-,CD45- }\end{array}$ & $\begin{array}{l}\text { CD9+,CD33+,CD13+ } \\
\text { CD15-,CD11- }\end{array}$ & $\begin{array}{c}17 p- \\
\text { CD45+,CD33t, } \\
\text { CD13+,CD15+, } \\
\text { CD34t,CD7- }\end{array}$ \\
\hline
\end{tabular}

PML-RARA $\alpha$

- Southernblot

- RT-PCR

Negativo

Positivo

Positivo

Positivo Positivo No realizado

No realizado

Positivo

Positivo

(*) CID: Coagulación intravascular diseminada. 
TABLA II

\section{ANIFESTACIO NES CLÍNICAS DEL SA Y RESPUESTA A TRATAM IENTO}

\begin{tabular}{|c|c|c|c|c|c|}
\hline & Paciente 1 & Paciente 2 & Paciente 3 & Paciente 4 & Paciente 5 \\
\hline $\begin{array}{l}\text { Status al } \\
\text { diaanóstico }\end{array}$ & De novo & De novo & De novo & Recidiva & Recidiva \\
\hline Tratamiento & $\begin{array}{c}\text { ATRA* } \\
\text { Ara-C/Dauno** } \\
\text { desde día }+1\end{array}$ & $\begin{array}{c}\text { ATRA* } \\
\text { Ara C/Dauno** } \\
\text { desde día }+2\end{array}$ & $\begin{array}{c}\text { ATRA* } \\
\text { AraC/ Dauno** } \\
\text { desde día }+2\end{array}$ & ATRA* & ATRA* \\
\hline Día inicio SA & +9 & +2 & +3 & +1 & +11 \\
\hline $\begin{array}{l}\text { Leucocitos al } \\
\text { inicio del SA } \\
\left(\times 10^{6} / \mathrm{mL}\right)\end{array}$ & 3,2 & 13,4 & 14,45 & 25,5 & 7,52 \\
\hline Clínica & $\begin{array}{c}\text { - Fiebre } \\
\text { - Insuf. respiratoria } \\
\text { - Alteración } \\
\text { bioquímica hepática }\end{array}$ & $\begin{array}{l}\text { - Fiebre } \\
\text { - Insuf. respiratoria } \\
\text { - Edemas }\end{array}$ & $\begin{array}{c}\text { - Fiebre } \\
\text { - Insuf. respiratoria }\end{array}$ & $\begin{array}{c}\text { - Fiebre } \\
\text { - Insuf. respiratoria } \\
\text { - Alteración bioquímica }\end{array}$ & $\begin{array}{c}\text { - Fiebre } \\
\text { - Insuf. respiratoria } \\
\text { hepática }\end{array}$ \\
\hline $\begin{array}{l}\text { Tratamiento } \\
\text { Dexametasona }\end{array}$ & $\mathrm{Si}$ & $\mathrm{Si}$ & $\mathrm{Si}$ & NO & $\mathrm{Si}$ \\
\hline $\begin{array}{l}5 \text { días } \\
\text { Evolución }\end{array}$ & $\begin{array}{c}10 \text { días } \\
\text { Remisión en } 24 \\
\text { horas }\end{array}$ & $\begin{array}{c}8 \text { días } \\
\text { Respuesta lenta } \\
7 \text { días }\end{array}$ & $\begin{array}{c}\text { M ejoría en } 24 \\
\text { horas }\end{array}$ & $\begin{array}{c}\text { M uerte en } 24 \\
\text { horas }\end{array}$ & M uerte en 48 horas \\
\hline
\end{tabular}

*ATRA 45 mg/m2/ día. **Daunorrubicina 60 mg/ m2/ día durante 3 días y Citarabina 200 mg/m2/ día durante 7 días.

\section{DISCUSIÓN}

El SA tal y como se ha descrito previamente, es una complicación característica del tratamiento de la LPA y una de las principales limitaciones del uso de ATRA. Hay una gran semejanza entre el SA y el síndrome de hiperpermeabilidad capilar. Este síndrome fue descrito tras la administración de citoquinas como la interleukina -2 (IL-2) (17) o el factor estimulante de colonias granulocito-macrofágicas (GM-CSF) $(18,19)$ y sus manifestaciones mas características son al igual que en el SA la fiebre, hipoxemia con infiltrados pulmonares, retención de líquidos y la progresión a disfunción multiorgánica. Posiblemente ambos cuadros obedecen a un mecanismo común, aunque el modo en que se desarrollan es todavía desconocido. Se ha propuesto la hipótesis de que el desarrollo del SA se debe a la activación leucocitaria inducida por el ATRA y mediada por liberación de citoquinas. La secuencia de acontecimientos sería dependiente de la diferenciación de las células leucémicas por efecto del ATRA, que se asocia a la liberación de las citoquinas vasoactivas interleukina 1 (IL-1) e interleukina 6 (IL-6) en la microcirculación, y junto al factor de necrosis tumoral (TNF$\alpha$ ), producen aumento de la permeabilidad capilar (20), a través de la generación de óxido nítrico (NO) (11). Alternativamente, el ATRA podría inducir en las células leucémicas en diferenciación, la adquisición de propiedades migratorias anómalas (16) o la expresión de integrinas (ICAM-1) y otras moléculas de adhesión que medien en el fracaso de la microcirculación. Así, la actividad de los esteroides en el control del SA se fundamentaría en su capacidad de inhibición de la formación del óxido nítrico y de la expresión de ICAM-1 (20,21).

Un síndrome clínico similar al SA también se ha observado en pacientes con LPA que no han sido tratados con ATRA (22), lo que podría indicar que el uso de ATRA potencia el defecto causal, que reside en el propio clon tumoral de la LPA $(17,23)$. Excepcionalmente, en otros tipos de leucemia y tumores sólidos tratados con ATRA se ha visto también un cuadro similar en asociación a leucocitosis o sin ella (24-27), indicando que el ATRA utilizado en otras patologías quizá pueda provocar un síndrome activación leucocitaria con consecuencias devastadoras en órganos clave.

En este trabajo describimos cinco casos de leucemia promielocítica aguda (LPA) que desarrollaron síntomas y signos compatibles con el síndrome de ATRA (SA), en el curso del tratamiento de inducción con ATRA en monoterapia o combinado con quimioterapia citotóxica. Las primeras manifestaciones comenzaron en el día uno a once desde el inicio del tratamiento, con fiebre en todos los casos, insuficiencia respiratoria con infiltrados pulmonares en la radiología y ganancia de peso sin evidencia de fallo cardíaco. Estos signos no son específicos indican edema pulmonar no cardiogénico. En el caso de la LPA tratada con ATRA, el diagnóstico de SA es muy sugestivo una vez descartada la presencia de infecciones graves, fallo cardíaco y otras reacciones pulmonares como la hemorragia alveolar y enfermedad tromboembólica. En nuestros pacientes descartamos razonablemente una infección no controlada como causa del cuadro, ya que los hemocultivos, cultivos de secreciones respiratorias y otros estudios microbiológicos fueron negativos a la presentación del síndrome. Antes de desarrollar el cuadro un paciente (Caso 5) había tenido un hemocultivo de $P$. Aerugi nosa y en todos los casos se administraron antibióticos de amplio espectro antes del inicio del SA o en su curso, sin evidencia de mejoría. Todos los casos presentaron ganancia de peso que no se justificaba por fallo cardíaco o sobrecarga aguda de volumen, ya que la presión venosa central se encontraba dentro del rango normal, no había signos pulmonares de edema a y los electrocardiogramas no presentaron alteraciones. Es 
más, en cuatro pacientes se realizaron ecocardiogramas 2D que mostraron una función cardiaca conservada. La restricción en el aporte de líquidos o el uso de diuréticos no fueron eficaces en controlar este cuadro clínico cuando se utilizaron antes de la introdución de los esteroides. Es de destacar que en dos casos hubo una neumonitis difusa con hemoptisis. En el caso $n^{\circ} 3$ la presentación del SA fue muy precoz y similar a una hemorragia alveolar difusa, y en el caso $n^{\circ} 5$ cuya evolución fue fatal no pudimos excluir completamente una infección oportunista.

Del análisis de esta serie extraemos que el SA puede aparecer en distintas fases de la LPA, durante el tratamiento inicial como en el curso de una recidiva y tanto el LPA de novo como en los casos con antecedente de otros tumores sólidos como el cáncer de mama. Así mismo, hemos visto que las dos variedades de LPA, hipergranular o microgranular pueden ser susceptibles de desarrollar el SA durante el tratamiento con ATRA.

Un aspecto interesante y no del todo explicado es, que si bien todos los pacientes aquí descritos cumplían los criterios diagnósticos de la clasificación FAB para el diagnóstico de la leucemia promielocítica aguda (28), esto es la presencia de blastos hipergranulares y promielocitos atípicos en cuantía superior al 30\%, únicamente en un caso encontramos la $\mathrm{t}(15 ; 17)$ típica y en cuatro el reordenamiento PML-RARAa incluido el anteriormente referido. En el paciente 1 se demostró el reordenamiento genético en la evolución posterior. Anteriormente se ha referido que el SA es una complicación exclusiva de la LPA (6) y uno de nuestros pacientes no cumplía los criterios genéticos de la LPA. No obstante, la presentación clínica con coagulopatía de consumo, y la morfología y citoquímica de los blastos junto al perfil de expresión de antígenos de diferenciación típico de la LPA, hacen que este caso sean indistinguible de otros diagnosticados en nuestro centro que sí cumplen los criterios genéticos de la LPA.

En esta serie también hemos observado que el SA fue de aparición más precoz en los casos en que se produjo un incremento en la cifra de leucocitos en el seno del tratamiento, y que el curso clínico fue claramente mejor en los pacientes con LPA de novo y en los que se estableció precozmente tratamiento con dexametasona. En tres de los cuatro casos tratados con dexametasona el cuadro se resolvió hasta la resolución sin secuelas. Los dos pacientes en los que el curso del SA fue rápidamente fatal presentaban LPA en recidiva, y habían presentado toxicidad importante por el tratamiento antileucémico previo. Quizá su mayor deterioro biológico contribuyó a precipitar el fallo multiorgánico.

Actualmente la mayoría de los regímenes de inducción de la LPA incluyen la administración de ATRA, y dada la transcendencia clínica del SA, diversos grupos han analizado el valor predictivo de las diversas características clínico-biológicas de la LPA y el riesgo de desarrollar el SA. Inicialmente se vio, como aquí hemos comprobado, que ni la diferente morfología hipergranular o variante, ni la presencia o severidad de la coagulopatía de consumo o la presencia de fiebre o infección a la presentación de la LPA determina un riesgo distinto de padecer el SA (29). Pero la relación entre una cifra elevada de leucocitos en sangre y una la mayor incidencia de SA es clara. Mas de la mitad de los casos de LPA que alcanzan cifras de leucocitos superiores a $20,0 \times 10^{9} / \mathrm{L}$ al diagnóstico o durante el tratamiento con ATRA desarrollan el SA $(7,16)$. El riesgo relativo de la leucocitosis es mayor si el incremento se produce en el seno del tratamiento con ATRA (10,29-32), mientras que la leucocitosis inicial, si no se acompaña de un incremento es menos determinante $(16,21,29)$. Globalmente, la leucocitosis es un factor predictivo de SA, con una sensibilidad entre un 44 y $62 \%$ y una especificidad entre 67 y $72 \%(29,30)$. Dos de nuestros pacientes presentaron el SA en presencia de cifras de leucocitos en aumento, uno al inicio del tratamiento y otro coincidiendo con la regeneración hemoperiférica. También se ha relacionado la expresión de la aminopeptidasa N (CD13) en células leucémicas de la APL, con una mayor frecuencia de SA (29). Esta aminopeptidasa interviene en la capacidad de invasión tisular de células tumorales $(33,34)$. Finalmente, la localización del punto de ruptura en los cromosomas 15 y 17, y las diferentes proteínas de fusión PMLRARAa no se han relacionado con el riesgo de SA $(7,29)$.

Distintas dosificaciones de ATRA (25 y $45 \mathrm{mg} / \mathrm{m}^{2} /$ día $)$ utilizadas en adultos con LPA, no han mostrado diferencias en la actividad antileucémica o la incidencia de SA (19), aunque se ha visto que la monoterapia con dosis de ATRA mas reducidas produce menos leucocitosis y menos casos de SA sin reducir el número de remisiones completas obtenidas (35).

Con todo, la estrategia utilizada habitualmente para prevenir el SA es la adición de quimioterapia dirigida a controlar la leucocitosis. La administración de hidroxiurea se ha utilizado con éxito (29), pero la mayoría de los grupos se han inclinado por utilizar quimioterapia convencional, con antraciclinas acompañadas o no de citarabina, en función de los recuentos de leucocitos al diagnóstico o en los días sucesivos de tratamiento con ATRA $(6,8,36,37)$. Esta medida reduce la incidencia de SA manteniendo una elevada tasa de remisiones completas, aunque la mielosupresión severa puede producir mas complicaciones infecciosas y hemorragias y aumentar los costes del tratamiento. Pero a largo plazo, la combinación de ATRA y quimioterapia en la inducción disminuye el número recidivas leucémicas $(12,38)$. Otra posibilidad es el uso profiláctico de esteroides, y el uso de prednisona oral, $75 \mathrm{mg} /$ día, desde el inicio del tratamiento en pacientes con leucocitosis superior a $10 \times 10^{9} / \mathrm{L}$ reduce el riesgo de SA sin afectar el índice de remisiones completas (21). En un futuro, el empleo de ATRA vehiculizado en liposomas puede tener un papel muy importante, ya que estudios preliminares sugieren que tiene un menor desarrollo de resistencias y menor incidencia de SA (39).

Una vez que se ha manifestado el SA, el único tratamiento eficaz es la administración precoz de esteroides en dosis altas, habitualmente dexametasona $10 \mathrm{mg} / 12 \mathrm{~h}$ durante un mínimo de 10 días $(16,29)$. En nuestra serie cuatro de los pacientes fueron tratados con esta pauta. Tres casos de LPA de nuevo diagnóstico tuvieron una buena respuesta, mientras que el caso de LPA en recidiva fue resistente y falleció, al igual que el otro caso que no se trató con esteroides. No se ha evaluado si el riesgo de fallecimiento por SA es mayor en pacientes con LPA en recidiva, aunque nuestra serie parece indicar un peor pronóstico, que como se ha comentado puede estar relacionado con un peor estado biológico. No se ha establecido que la retirada del ATRA al inicio del SA sea una medida eficaz $(1,16)$, aunque se considera prudente interrumpir su administración hasta que los síntomas y signos del SA hayan remitido. La adición de quimioterapia una vez establecido el SA o la realización de leucoféresis no contribuyen al control del síndrome $(12,16,29,31)$.

\section{CONCLUSIONES}

El SA es una complicación frecuente y grave del tratamiento de la LPA con ATRA, con un elevado riesgo de muerte por insuficiencia respiratoria y fallo multiorgánico. Es esencial reconocer rápidamente el cuadro pues el tratamiento precoz con dexametasona es efectivo en muchos casos. 


\section{Bibliografía}

1. Warrell R P, De The H, Nang ZY, Degos L. Acute promyelocyte leukemia. N. Engl J Med 1993; 329: 177-189.

2. Grignani F, Fagioli M, Alcalay M, Longo L, Rudolfi P, Donti E et al. Acute promyelocytic leukemia: from genetics to treatment. Blood 1994; 83: $10-25$.

3. Sanz MA, Jarque I, Martin G, Lorenzo I, Martinez J, Rafecas J, et al. Acute promyelocytic leukemia. Therapy results and prognostic factors. Cancer 1988; 61: 7-13

4. Kantarjian HM, Keating MJ, Walters RS, Smith TL, McCredie KB, Freireich EJ. Role of maintenance chemotherapy in acute promyelocytic leukemia. Cancer 1987; 59: 1258-1263

5. Head D, Kopecky KJ, Weick J, Files JC, Ryan D, Foucar K, et al Effect of aggressive daunomycin therapy on survival in acute promyelocytic leukemia. Blood 1995; 86: 1717-1728

6. Fenaux P, Le Deley MC, Castaigne S, Archimbaud E, Chomienne C, Linr $\mathrm{H}$ et al. European APL 91 Group. Effect of all transretinoic acid in neuwly diagnosed acute promyelocytic leukemia. Results of a multicenter randomised trial. Blood 1993; 82: 3241-3249.

7. Frankel SR, Eardley A, Heller G, Berman E, Miller W Dmitrovsky E et al. All-transrenoic acid for the treatment of newly diagnosed acute promyelocytic leukemia. Result of the New York Study. Ann Intern Med 1994; 120: 278-286.

8. Ranamara A, Takemoto Y, Tanimoto M, Hurakami H, Asou N, Robayashi $\mathrm{T}$ et al. All-trans retinoic acid for the treatment of newly diagnosed acute promyelocytic leukemia. Blood 1995; 85: 1202-1206.

9. Chen ZX, Xue YQ, Zhang R, Tao RF, Yia YM, et al. A clinical and experimental study on all-trans retinoic acid treated acute promyelocytic leukemia patients. Blood 78: 1413-1419.

10. Fenaux $\mathrm{P}$ and the APL group. Treatment of newly diagnosed APL. The best choice is not ATRA or chemoterapy... but a combination of both. Leukemia 1994; 8: S59-S61.

11. Fenaux P, Chastany C, Chomienne C, Castaigne S, Sanz M, Link H et al. Treatment of newly diagnosed acute promyelocytic leukemia (APL) by all transretinoic acid (ATRA) combined with chemotherapy: The European Experience. Leuk Lymphoma 1995; 16: 431-437.

12. Tallman MS, Andersen JW, Schiffer CA, Appelbaum FR, Feusner JH, Ogden A, Shepherd L, Willman C, Bloomfield CD, Rowe JM, Wiernik PHN. All-trans-retinoic acid in acute promyelocytic leukemia. N Engl J Med 1997; 337: 1021-1028

13. Shen ZX, Chen GQ, Ni JH, Li XS, Xiong SM, Qiu QY, et al. Use of arsenic trioxide (As2O3) in the treatment of acute promyelocytic leukemia (APL): II. Clinical efficacy and pharmacokinetics in relapsed patients.Blood $1992 ; 89$ : 3354-3360

14. Muindi J, Frankel SR, Miller WH Jr, Jakubowski A, Scheinberg DA, Young $\mathrm{CW}$, et al. Continuous treatment with all-trans retinoic acid causes a progressive reduction in plasma drug concentrations: implications for relapse and retinoid "resistance" in patients with acute promyelocytic leukemia.Blood 1992; 79: 299-303

15. Adamson PC, Boylan JF, Balis FM, Murphy RF, Godwin KA, Gudas LJ, Poplack DG.Time course of induction of metabolism of all-transretinoic acid and thup-regulation of cellular retinoic acid-binding protein.Cancer Res 1993; 53: 472-476

16. Frankel SR, Eardley A, Lauwers G, Neiss M, Narrel R P. The "retinoic acid syndrome" in acute promyelocytic leukemia. Ann Intern Med 1992; 117: 292-246.

17. Margotin RA, Ruyner AA, Hawkins HJ, Atkins MB, Dutcher J P, Fhiser RI et al. Interleukin-2 and lymphokine-activated killer cell therapy of solid tumors; analysis of toxicity and mangement guidelines. J Clin Oncol 1989; 7: 486-498.

18. Niley J S. Citokine priming on acute myelocytic leukemia may produce a pulmonare syndrome when associated with a rapid increase in peripheral blood myeloblasts. Blood 1993; 82: 3511-3512.

19. Drombet H, Castaigne S, Fenaux P, Chomienne, Degos L. Induction treatment of acute promyelocytic leukemia using all-trans retinoic acid. Controversia about dosage, advantages and side effects mana- gement. Leukemia 1994; 8: 73-S75.

20. Languino LR, Plescia J, Dupernay A, Brian AA, Plow EF, Geltosr JE et al. Fibrinogen mediates leukocyte adhesion to vascular endothelium through an ICAM-1 dependent pathway. Cell 1993; 73: 1423-1434.

21. Nileg JS, Firkin FC. Reduction of pulmonary toxicity by prednisolone prophilaxis during all-trans retinoic acid treatment of acute promyelocytic leukaemia. Australian Leukemia Study Group. Leukemia 1995; 9: 774-778.

22. Stadler M, Ganser A, Hoelzer D. Acute promyelocytic leukemia. N Engl J Med 1994; 330: 140-141.

23. Warrel RP Jr. Fatal all-trans retinoic acid pneumonitis. Ann Intern Med 1993; $118: 473$.

24. Mahmoud H Z, Hurwitz C A, Roberts W M, Santana V M, Ribeiro R C, Krance $\mathrm{R}$ A. Tretinoin toxicity in children with acute promyelocytic leukaemia. Lancet 1993; 342: 1394-1395.

25. Kahn MJ, Luginbuhl W, Gaines L. Leukocytosis associated with alltrans retinoic acid therapy in metastatic non small-cell lung cancer. J Natl Cancer Inst 1992: 84: 1669-1671.

26. Kurzrock R, Estey E, Talpaz M. All-trans retinoic acid tolerance and biologic effects in myelodysplastic syndrome. J Clin Oncol 1993; 11 : 1489-1495.

27. Cortes JE, Kantargian H, O'Brien S, Robertson LE, Koller C. HirshGinsberg et al. All-trans retinoic acid followed by chemotherapy for salvage of refractory or relapse acute promyelocytic leukemia. Cancer 1994; 73: 2946-2952.

28. Bennet JM, Catovsky D, Daniel M T, Aundrin G, Galton DA, Gralnick H.R. et al. Proposed revised criteria for the classification of acute myeloid leukemia: a report of the French-American-British cooperative group. Ann Intern Med 1985; 103: 620-625.

29. Vahdat L, Maslak P, Miller NH Jr, Eardley A, Heller G, Scheinberg DA. et al. Early mortality and the retinoic acid syndrome in acute promyelocytic leukemia: Impact of leukocytosis low-dose chemotherapy, PMN/RARA-a isoform, and CD13 expression in patients treated with all-trans retinoic acid. Blood 1994; 84: 3843-3849.

30. Cambier N, Wattel E, Menot M L, Guerci A, Chomiennec C, Fenaux P All trans retinoic acid chronic myelomonocytic leukemia, results of a pilot study. Leukemia 1996; 10: 1164-7.

31. Castangue S, Degos L. Treatment of acute promyelocytic leukemia by all trans retinoic acid. C.R. Sceances Soc. Biol Fil. 1995; 189: 515-20.

32. Sun GL. Follow-up of 524 patients with APL after CR induces by ATRA. International Symposium on retinoids in Hematologic Malignancies, Shangai, China. 1994; 8: 1074-1091.

33. Menrad A, Speicher D, Wacker J, Herlyn M. Biochemical and functional characteritation of aminopeptidase $\mathrm{N}$ expressed by human melanoma cells. Cancer Res 1993; 53: 1450-1454.

34. Saiki I, Fuji H, Yoneda J, Abe F, Nakajima M, Tsurmo F. et al. Role of aminopeptidase $\mathrm{N}$ (cd13) in tumor cell invasion and extracellular matrix degeneration. Int J Cancer 1993; 54: 137-142.

35. Chen CQ, Shen ZX, Wu F, Han JY, Miao JM, Zhong HJ, et al. Pharmacokinetics and efficacy of low dose all trans retinoic acid in the treatment of acute promyelocytic leukemia.Leukemia 1996; 10: 825-8.

36. Fenaux P. Results of APL 91 European Trial combining ATRA and chemotherapy. Presentation of APL 1993 Trial. Leukemia 1994; 8: 70-72.

37. Fenaux P, Chastang C, Degos L and French APL Group. Treatment of promyelocytic leukemia (APL) by a combination of all trans-trans retinoic acid (ATRA) and chemotherapy. Leukemia 1994; 8: S42-47.

38. Fenaux P, Chastang C, Degos L and the European APL Group. Treatment of promyelocytic leukemia (APL) by a combination of all trans-trans retinoic acid (ATRA) and chemotherapy. Blood 1997; 90: 333 .

39. Estey E, Thall PF, Mehta K, Rosemblum M, Breuer T, Simmons V, et al. Alterations in tretinoin pharmacokinetics following administration of liposomal all trans retinoic acid. Blood 1996; 87: 3650-3655.nal characteritation of aminopeptidase $\mathrm{N}$ expressed by human melanoma cells. Cancer Res 1993; 53: 1450-1454. 